\title{
Prefix and Right-Partial Derivative Automata *
}

\author{
Eva Maia, Nelma Moreira, Rogério Reis \\ CMUP \& DCC, Faculdade de Ciências da Universidade do Porto \\ Rua do Campo Alegre, 4169-007 Porto, Portugal \\ e-mail:\{emaia, nam,rvr\}@dcc.fc.up.pt
}

\begin{abstract}
Recently, Yamamoto presented a new method for the conversion from regular expressions (REs) to non-deterministic finite automata (NFA) based on the Thompson $\varepsilon$-NFA $\left(\mathcal{A}_{\mathrm{T}}\right)$. The $\mathcal{A}_{\mathrm{T}}$ automaton has two quotients discussed: the suffix automaton $\mathcal{A}_{\text {suf }}$ and the prefix automaton, $\mathcal{A}_{\text {pre }}$. Eliminating $\varepsilon$-transitions in $\mathcal{A}_{\mathrm{T}}$, the Glushkov automaton $\left(\mathcal{A}_{\text {pos }}\right)$ is obtained. Thus, it is easy to see that $\mathcal{A}_{\text {suf }}$ and the partial derivative automaton $\left(\mathcal{A}_{\mathrm{pd}}\right)$ are the same. In this paper, we characterise the $\mathcal{A}_{\text {pre }}$ automaton as a solution of a system of left $\mathrm{RE}$ equations and express it as a quotient of $\mathcal{A}_{\text {pos }}$ by a specific left-invariant equivalence relation. We define and characterise the right-partial derivative automaton $\left(\overleftarrow{\mathcal{A}}_{\mathrm{pd}}\right)$ Finally, we study the average size of all these constructions both experimentally and from an analytic combinatorics point of view.
\end{abstract}

\section{Introduction}

Conversion methods from regular expressions to equivalent nondeterministic finite automata have been widely studied. Resulting NFAs can have $\varepsilon$-transitions or not. The standard conversion with $\varepsilon$-transitions is the Thompson automaton $\left(\mathcal{A}_{\mathrm{T}}\right)[15]$ and the standard conversion without $\varepsilon$-transitions is the Glushkov (or position) automaton $\left(\mathcal{A}_{\text {pos }}\right)[9]$. Other conversions such as partial derivative automaton $\left(\mathcal{A}_{\mathrm{pd}}\right)[1,13]$ or follow automaton $\left(\mathcal{A}_{\mathrm{f}}\right)[10]$ were proved to be quotients of the $\mathcal{A}_{\text {pos }}$, by specific right-invariant equivalence relations $[6,10]$. In particular, for REs under special conditions, $\mathcal{A}_{\mathrm{pd}}$ is an optimal conversion method [12]. Moreover, asymptotically and on average, the size of $\mathcal{A}_{\mathrm{pd}}$ is half the size of $\mathcal{A}_{\text {pos }}$ [3]. Reductions on the size of NFAs using left-relations was studied recently by Ko and Han [11].

Yamamoto [16] presented a new conversion method based on the $\mathcal{A}_{\mathrm{T}}$. Given a $\mathcal{A}_{\mathrm{T}}$, two automata are constructed by merging $\mathcal{A}_{\mathrm{T}}$ states: in one, the suffix automaton $\left(\mathcal{A}_{\text {suf }}\right)$, states with the same right languages and in the other, the prefix automaton $\left(\mathcal{A}_{\text {pre }}\right)$, states with the same left languages. $\mathcal{A}_{\text {suf }}$ corresponds to $\mathcal{A}_{\mathrm{pd}}$, which is not a surprise because it is known that if $\varepsilon$-transitions are eliminated from $\mathcal{A}_{\mathrm{T}}$, the $\mathcal{A}_{\text {pos }}$ is obtained [8]. $\mathcal{A}_{\text {pre }}$ is a quotient by a left-invariant

\footnotetext{
* This work was partially funded by the European Regional Development Fund through the programme COMPETE and by the Portuguese Government through the FCT under project UID/MAT/00144/2013 and project FCOMP-01-0124-FEDER020486. Eva Maia was also funded by FCT grant SFRH/BD/78392/2011.
} 
relation. In this paper, we further study conversions from REs to NFAs based on left-invariant relations. Using the notion of right-partial derivatives introduced by Champarnaud et. al [4], we define the right-partial derivative automaton $\overleftarrow{\mathcal{A}}_{\text {pd }}$, characterise its relation with $\mathcal{A}_{\text {pd }}$ and $\mathcal{A}_{\text {pos }}$, and study its average size. We construct the $\mathcal{A}_{\text {pre }}$ automaton directly from a regular expression without use the $\mathcal{A}_{\mathrm{T}}$ automaton, and we show that it is also a quotient of the $\mathcal{A}_{\text {pos }}$. However, the experimental results suggest that, on average, the reduction on the size of the $\mathcal{A}_{\text {pos }}$ is not large. Considering the framework of analytic combinatorics we study this reduction.

\section{Regular Expressions and Automata}

Given an alphabet $\Sigma=\left\{\sigma_{1}, \sigma_{2}, \ldots, \sigma_{k}\right\}$ of size $k$, the set RE of regular expressions $\alpha$ over $\Sigma$ is defined by the following grammar:

$$
\alpha:=\emptyset|\varepsilon| \sigma_{1}|\cdots| \sigma_{k}|(\alpha+\alpha)|(\alpha \cdot \alpha) \mid(\alpha)^{\star},
$$

where the $\cdot$ is often omitted. If two REs $\alpha$ and $\beta$ are syntactically equal, we write $\alpha \sim \beta$. The size of a $\operatorname{RE} \alpha,|\alpha|$, is its number of symbols, disregarding parenthesis, and its alphabetic size, $|\alpha|_{\Sigma}$, is the number of occurrences of letters from $\Sigma$. A $\mathrm{RE} \alpha$ is linear if all its letters occurs only once. The language represented by a RE $\alpha$ is denoted by $\mathcal{L}(\alpha)$. Two REs $\alpha$ and $\beta$ are equivalent if $\mathcal{L}(\alpha)=\mathcal{L}(\beta)$, and we write $\alpha=\beta$. We define the function $\varepsilon$ by $\varepsilon(\alpha)=\varepsilon$ if $\varepsilon \in \mathcal{L}(\alpha)$ and $\varepsilon(\alpha)=\emptyset$, otherwise. This function can be naturally extended to sets of REs and languages. We consider REs reduced by the following rules: $\varepsilon \alpha=\alpha=\alpha \varepsilon$, $\emptyset+\alpha=\alpha=\alpha+\emptyset$, and $\emptyset \alpha=\emptyset=\alpha \emptyset$. Given a language $\mathcal{L} \subseteq \Sigma^{\star}$ and a word $w \in \Sigma^{\star}$, the left quotient of $\mathcal{L}$ w.r.t. $w$ is the language $w^{-1} L=\{x \mid w x \in L\}$, and the right quotient of $\mathcal{L}$ w.r.t. $w$ is the language $\mathcal{L} w^{-1}=\{x \mid x w \in \mathcal{L}\}$. The reversal of a word $w=\sigma_{1} \sigma_{2} \cdots \sigma_{n}$ is $w^{R}=\sigma_{n} \cdots \sigma_{2} \sigma_{1}$. The reversal of a language $\mathcal{L}$, denoted by $\mathcal{L}^{R}$, is the set of words whose reversal is on $\mathcal{L}$. The reversal of $\alpha \in \mathrm{RE}$ is denoted by $\alpha^{R}$. The reversal of set of REs is the set of the reversal of its elements. It is not difficult to verify that $\mathcal{L} w^{-1}=\left(\left(w^{R}\right)^{-1} L^{R}\right)^{R}$.

A nondeterministic finite automaton (NFA) is a five-tuple $A=(Q, \Sigma, \delta, I, F)$ where $Q$ is a finite set of states, $\Sigma$ is a finite alphabet, $I \subseteq Q$ is the set of initial states, $F \subseteq Q$ is the set of final states, and $\delta: Q \times \Sigma \rightarrow 2^{Q}$ is the transition function. The transition function can be extended to words and to sets of states in the natural way. When $I=\left\{q_{0}\right\}$, we use $I=q_{0}$. Given a state $q \in Q$, the right language of $q$ is $\mathcal{L}_{q}(A)=\left\{w \in \Sigma^{\star} \mid \delta(q, w) \cap F \neq \emptyset\right\}$, and the left language is $\overleftarrow{\mathcal{L}}_{q}(A)=\left\{w \in \Sigma^{\star} \mid q \in \delta(I, w)\right\}$. The language accepted by $A$ is $\mathcal{L}(A)=\bigcup_{q \in I} \mathcal{L}_{q}(A)$. Two NFAs are equivalent if they accept the same language. If two NFAs $A$ and $B$ are isomorphic, we write $A \simeq B$. An NFA is deterministic if for all $(q, \sigma) \in Q \times \Sigma,|\delta(q, \sigma)| \leq 1$ and $|I|=1$. The reversal of an automaton $A$ is the automaton $A^{R}$, where the sets of initial and final states are swapped and all transitions are reversed. Given an equivalence relation $\equiv$ in $Q$, the quotient automaton $A / \equiv=(Q / \equiv, \Sigma, \delta / \equiv, I / \equiv, F / \equiv)$ is defined in the usual 
way. A relation $\equiv$ is right invariant w.r.t. $A$ if and only if: $\equiv \subseteq(Q-F)^{2} \cup F^{2}$ and $\forall p, q \in Q, \sigma \in \Sigma$, if $p \equiv q$, then $\delta(p, \sigma) / \equiv=\delta(q, \sigma) / \equiv$. A relation $\equiv$ is a left invariant relation w.r.t. $A$ if and only if it is a right-invariant relation w.r.t. $A^{R}$.

The right languages $\mathcal{L}_{i}$, for $i \in Q=[0, n]$, define a system of right equations, $\mathcal{L}_{i}=\bigcup_{j=1}^{k} \sigma_{j}\left(\bigcup_{m \in I_{i j}} \mathcal{L}_{m}\right) \cup \varepsilon\left(\mathcal{L}_{i}\right)$, where $I_{i j} \subseteq[0, n], m \in I_{i j} \Leftrightarrow m \in \delta\left(i, \sigma_{j}\right)$, and $\mathcal{L}(A)=\bigcup_{i \in I} \mathcal{L}_{i}$. In the same manner, the left languages of the states of $A$ define a system of left equations $\overleftarrow{\mathcal{L}}_{i}=\bigcup_{j=1}^{k}\left(\bigcup_{m \in I_{i j}} \overleftarrow{\mathcal{L}}_{m}\right) \sigma_{j} \cup \varepsilon\left(\overleftarrow{\mathcal{L}}_{i}\right)$, where $I_{i j} \subseteq[0, n], m \in I_{i j} \Leftrightarrow i \in \delta\left(m, \sigma_{j}\right)$, and $\mathcal{L}(A)=\bigcup_{i \in F} \overleftarrow{\mathcal{L}}_{i}$

\subsection{Glushkov and Partial Derivative Automata}

In the following we review two constructions which define NFAs equivalent to a given regular expression $\alpha \in \mathrm{RE}$. Let $\operatorname{pos}(\alpha)=\left\{1,2, \ldots,|\alpha|_{\Sigma}\right\}$ be the set of letter positions in $\alpha$, and let $\operatorname{pos}_{0}(\alpha)=\operatorname{pos}(\alpha) \cup\{0\}$. We consider the expression $\bar{\alpha}$ obtained by marking each letter with its position in $\alpha$, i.e. $\mathcal{L}(\bar{\alpha}) \in \bar{\Sigma}^{\star}$ where $\bar{\Sigma}=\left\{\left.\sigma_{i}|\sigma \in \Sigma, 1 \leq i \leq| \alpha\right|_{\Sigma}\right\}$. The same notation is used to remove the markings, i.e., $\overline{\bar{\alpha}}=\alpha$. For $\alpha \in \operatorname{RE}$ and $i \in \operatorname{pos}(\alpha)$, let first $(\alpha)=\{i \mid \exists w \in$ $\left.\bar{\Sigma}^{\star}, \sigma_{i} w \in \mathcal{L}(\bar{\alpha})\right\}$, last $(\alpha)=\left\{i \mid \exists w \in \bar{\Sigma}^{\star}, w \sigma_{i} \in \mathcal{L}(\bar{\alpha})\right\}$ and follow $(\alpha, i)=\{j \mid$ $\left.\exists u, v \in \bar{\Sigma}^{\star}, u \sigma_{i} \sigma_{j} v \in \mathcal{L}(\bar{\alpha})\right\}$. The Glushkov automaton (or position automaton) for $\alpha$ is $\mathcal{A}_{\text {pos }}(\alpha)=\left(\operatorname{pos}_{0}(\alpha), \Sigma, \delta_{\text {pos }}, 0, F\right)$, with $\delta_{\text {pos }}=\left\{\left(0, \overline{\sigma_{j}}, j\right) \mid j \in \operatorname{first}(\alpha)\right\} \cup$ $\left\{\left(i, \overline{\sigma_{j}}, j\right) \mid j \in\right.$ follow $\left.(\alpha, i)\right\}$ and $F=\operatorname{last}(\alpha) \cup\{0\}$ if $\varepsilon(\alpha)=\varepsilon$, and $F=\operatorname{last}(\alpha)$, otherwise. We note that the number of states of $\mathcal{A}_{\text {pos }}(\alpha)$ is exactly $|\alpha|_{\Sigma}+1$.

The partial derivative automaton of a regular expression was introduced independently by Mirkin [13] and Antimirov [1]. Champarnaud and Ziadi [5] proved that the two formulations are equivalent. For a regular expression $\alpha \in \mathrm{RE}$ and a symbol $\sigma \in \Sigma$, the set of left-partial derivatives of $\alpha$ w.r.t. $\sigma$ is defined inductively as follows:

$$
\begin{aligned}
& \partial_{\sigma}(\emptyset)=\partial_{\sigma}(\varepsilon)=\emptyset \quad \partial_{\sigma}(\alpha+\beta)=\partial_{\sigma}(\alpha) \cup \partial_{\sigma}(\beta)
\end{aligned}
$$

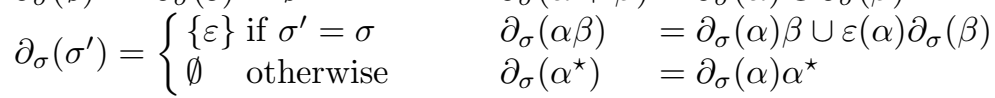

where for any $S \subseteq \mathrm{RE}, S \emptyset=\emptyset S=\emptyset, S \varepsilon=\varepsilon S=S$, and $S \beta=\{\alpha \beta \mid \alpha \in S\}$ if $\beta \neq \emptyset, \varepsilon$ (and analogously for $\beta S$ ). The definition of left-partial derivatives can be extended in a natural way to sets of regular expressions, words, and languages. We have that $w^{-1} \mathcal{L}(\alpha)=\mathcal{L}\left(\partial_{w}(\alpha)\right)=\bigcup_{\tau \in \partial_{w}(\alpha)} \mathcal{L}(\tau)$, for $w \in \Sigma^{\star}$. The set of all partial derivatives of $\alpha$ w.r.t. words is denoted by $\operatorname{PD}(\alpha)=\partial_{\Sigma^{\star}}(\alpha)$. The partial derivative automaton of $\alpha$ is $\mathcal{A}_{\mathrm{pd}}(\alpha)=\left(\mathrm{PD}(\alpha), \Sigma, \delta_{p d}, \alpha, F_{p d}\right)$, where $\delta_{p d}=$ $\left\{\left(\tau, \sigma, \tau^{\prime}\right) \mid \tau \in \operatorname{PD}(\alpha), \sigma \in \Sigma, \tau^{\prime} \in \partial_{\sigma}(\tau)\right\}$ and $F_{p d}=\{\tau \in \operatorname{PD}(\alpha) \mid \varepsilon(\tau)=\varepsilon\}$.

As noted by Broda et al. [3] and Maia et al. [12], following Mirkin's construction, the partial derivative automaton of $\alpha$ can be inductively constructed. A (right) support for $\alpha$ is a set of regular expressions $\left\{\alpha_{1}, \ldots, \alpha_{n}\right\}$ such that $\alpha_{i}=\sigma_{1} \alpha_{i 1}+\cdots+\sigma_{k} \alpha_{i k}+\varepsilon\left(\alpha_{i}\right), i \in[0, n], \alpha_{0} \sim \alpha$ and $\alpha_{i j}$ is a linear combination of $\alpha_{l}, l \in[1, n]$ and $j \in[1, k]$. The set $\pi(\alpha)$ inductively defined below is a 
right support of $\alpha$.

$$
\begin{aligned}
\pi(\emptyset) & =\emptyset & \pi(\alpha+\beta) & =\pi(\alpha) \cup \pi(\beta) \\
\pi(\varepsilon) & =\emptyset & \pi(\alpha \beta) & =\pi(\alpha) \beta \cup \pi(\beta) \\
\pi(\sigma) & =\{\varepsilon\} & \pi\left(\alpha^{\star}\right) & =\pi(\alpha) \alpha^{\star} .
\end{aligned}
$$

Champarnaud and Ziadi proved that $\operatorname{PD}(\alpha)=\pi(\alpha) \cup\{\alpha\}$ and the transition function of $\mathcal{A}_{\mathrm{pd}}$ can also be defined inductively from the system of equations above. Let $\varphi(\alpha)=\left\{(\sigma, \gamma) \mid \gamma \in \partial_{\sigma}(\alpha), \sigma \in \Sigma\right\}$ and $\lambda(\alpha)=\left\{\alpha^{\prime} \mid \alpha^{\prime} \in \pi(\alpha), \varepsilon\left(\alpha^{\prime}\right)=\varepsilon\right\}$, where both sets can be inductively defined using (2) and (3). We have, $\delta_{p d}=$ $\{\alpha\} \times \varphi(\alpha) \cup F(\alpha)$ where the result of the $\times$ operation is seen as a set of triples and the set $F$ is defined inductively by:

$$
\begin{aligned}
F(\emptyset) & =F(\varepsilon)=F(\sigma)=\emptyset, \sigma \in \Sigma \\
F(\alpha+\beta) & =F(\alpha) \cup F(\beta) \\
F(\alpha \beta) & =F(\alpha) \beta \cup F(\beta) \cup \lambda(\alpha) \beta \times \varphi(\beta) \\
F\left(\alpha^{\star}\right) & =F(\alpha) \alpha^{\star} \cup(\lambda(\alpha) \times \varphi(\alpha)) \alpha^{\star} .
\end{aligned}
$$

Note that the concatenation of a transition $(\alpha, \sigma, \beta)$ with a $\mathrm{RE} \gamma$ is defined by $(\alpha, \sigma, \beta) \gamma=(\alpha \gamma, \sigma, \beta \gamma)$ (similarly $\gamma(\alpha, \sigma, \beta)=(\gamma \alpha, \sigma, \gamma \beta))$, if $\gamma \notin\{\emptyset, \varepsilon\}$, $(\alpha, \sigma, \beta) \emptyset=\emptyset$ and $(\alpha, \sigma, \beta) \varepsilon=(\alpha, \sigma, \beta)$. Then, $\mathcal{A}_{\mathrm{pd}}(\alpha)=(\pi(\alpha) \cup\{\alpha\}, \Sigma,\{\alpha\} \times$ $\varphi(\alpha) \cup F(\alpha), \alpha, \lambda(\alpha) \cup \varepsilon(\alpha)\{\alpha\})$. In Fig. 1 are represented $\mathcal{A}_{\text {pos }}(\alpha)$ and $\mathcal{A}_{\mathrm{pd}}(\alpha)$, where $\alpha=\beta b$ and $\beta=\left(a^{\star} b+a^{\star} b a+a^{\star}\right)^{\star}$.

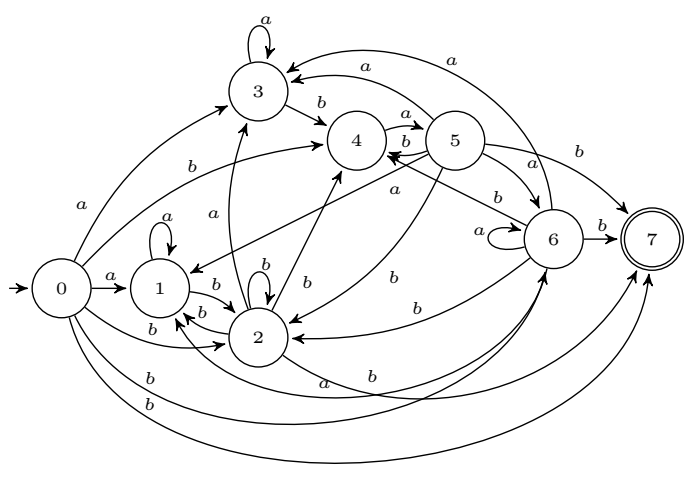

(a) $\mathcal{A}_{\text {pos }}(\alpha)$

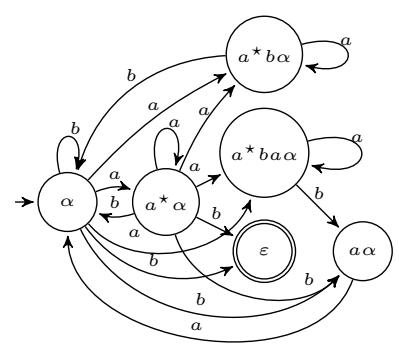

(b) $\mathcal{A}_{\mathrm{pd}}(\beta b)$

Fig. 1. Automata for $\alpha=\beta b$ with $\beta=\left(a^{\star} b+a^{\star} b a+a^{\star}\right)^{\star}$.

Champarnaud and Ziadi [6] showed that the partial derivative automaton is a quotient of the Glushkov automaton by the right-invariant equivalence relation $\equiv_{c}$, such that $i \equiv_{c} j$ if $\partial_{w \sigma_{i}}(\bar{\alpha})=\partial_{w \sigma_{j}}(\bar{\alpha})$, for $i, j \in \operatorname{pos}_{0}(\alpha)$ and let $\sigma_{0}=\varepsilon$. It is known that $\partial_{w \sigma_{i}}(\bar{\alpha})$ is either empty or an unique singleton for all $w \in \bar{\Sigma}^{\star}$. 


\section{Right-Partial Derivative Automata}

The concept of right-partial derivative was introduced by Champarnaud et. al. For a regular expression $\alpha \in \mathrm{RE}$ and a symbol $\sigma \in \Sigma$, the set of right-partial derivatives of $\alpha$ w.r.t. $\sigma, \overleftarrow{\partial}_{\sigma}(\alpha)$, is defined in the same way as the set of leftpartial derivatives except for the following two rules:

$$
\overleftarrow{\partial}_{\sigma}(\alpha \beta)=\alpha \overleftarrow{\partial}_{\sigma}(\beta) \cup \varepsilon(\beta) \overleftarrow{\partial}_{\sigma}(\alpha) \quad \overleftarrow{\partial}_{\sigma}\left(\alpha^{\star}\right)=\alpha^{\star} \overleftarrow{\partial}_{\sigma}(\alpha)
$$

This definition can be extended in a natural way to sets of regular expressions, words, and languages. The set of all right-partial derivatives of $\alpha$ w.r.t. words is denoted by $\overleftarrow{\mathrm{PD}}(\alpha)=\overleftarrow{\partial}_{\Sigma^{\star}}(\alpha)$. The right- and left-partial derivatives of $\alpha$ w.r.t. $w \in \Sigma^{\star}$ are related by $\overleftarrow{\partial}_{w}(\alpha)=\left(\partial_{w^{R}}\left(\alpha^{R}\right)\right)^{R}$. Thus, $\mathcal{L}\left(\overleftarrow{\partial}_{w}(\alpha)\right)=\mathcal{L}(\alpha) w^{-1}$. The right-partial derivative automaton of $\alpha, \overleftarrow{\mathcal{A}}_{\mathrm{pd}}(\alpha)$, can be defined inductively as a solution of a left system of expression equations, $\alpha_{i}=\alpha_{i 1} \sigma_{1}+\cdots+\alpha_{i k} \sigma_{k}+\varepsilon\left(\alpha_{i}\right)$, $i \in[0, n], \alpha_{0} \sim \alpha, \alpha_{i j}$ is a linear combination of $\alpha_{l}, l \in[1, n]$ and $j \in[1, k]$.

Proposition 1. The set of regular expressions $\overleftarrow{\pi}(\alpha)$ defined in the same way as the set $\pi$, except for the concatenation and Kleene star rules, is a solution of a left system of expression equations,

$$
\overleftarrow{\pi}(\alpha \beta)=\alpha \overleftarrow{\pi}(\beta) \cup \overleftarrow{\pi}(\alpha) \quad \overleftarrow{\pi}\left(\alpha^{\star}\right)=\alpha^{\star} \overleftarrow{\pi}(\alpha)
$$

Again, the solution of the system of equations also allows to inductively define the transition function. Let $\overleftarrow{\varphi}(\alpha)=\left\{(\gamma, \sigma) \mid \gamma \in \overleftarrow{\partial}_{\sigma}(\alpha), \sigma \in \Sigma\right\}$ and $\overleftarrow{\lambda}(\alpha)=$ $\left\{\alpha^{\prime} \mid \alpha^{\prime} \in \overleftarrow{\pi}(\alpha), \varepsilon\left(\alpha^{\prime}\right)=\varepsilon\right\}$, where both sets can be inductively defined using (5) and (6). The set of transitions of $\overleftarrow{\mathcal{A}}_{\mathrm{pd}}(\alpha)$ is $\overleftarrow{\varphi}(\alpha) \times\{\alpha\} \cup \overleftarrow{F}(\alpha)$ and the set $\overleftarrow{F}(\alpha)$ is defined similarly to the set $F(\alpha)$ except for the two following rules:

$$
\begin{aligned}
& \overleftarrow{F}(\alpha \beta)=\alpha \overleftarrow{F}(\beta) \cup \overleftarrow{F}(\alpha) \cup \varphi(\alpha) \times(\alpha \overleftarrow{\lambda}(\beta)) \\
& \overleftarrow{F}\left(\alpha^{\star}\right)=\alpha^{\star} \overleftarrow{F}(\alpha) \cup \alpha^{\star}(\overleftarrow{\varphi}(\alpha) \times \overleftarrow{\lambda}(\alpha))
\end{aligned}
$$

The right-partial derivative automaton of $\alpha$ is $\overleftarrow{\mathcal{A}}_{\mathrm{pd}}(\alpha)=(\overleftarrow{\pi}(\alpha) \cup\{\alpha\}, \Sigma, \overleftarrow{\varphi}(\alpha) \times$ $\{\alpha\} \cup \overleftarrow{F}(\alpha), \overleftarrow{\lambda}(\alpha) \cup \varepsilon(\alpha)\{\alpha\},\{\alpha\})$. In Fig. 3(a) is represented the $\overleftarrow{\mathcal{A}}_{\text {pd }}$ of the RE $\beta b$ considered in Fig. 1. Note that the sizes of $\pi(\alpha)$ and $\overleftarrow{\pi}(\alpha)$ are not comparable in general. For example, $|\pi(\beta b)|>|\overleftarrow{\pi}(\beta b)|$, but if we consider $\alpha=b\left(b a^{\star}+a b a^{\star}+\right.$ $\left.a^{\star}\right)^{\star}$ then $|\pi(\alpha)|<|\overleftarrow{\pi}(\alpha)|$. The following result relates the functions defined above to the ones used to define the $\mathcal{A}_{\mathrm{pd}}$ is given by the following result.

Proposition 2. Let $\alpha$ be a regular expression. Then $\overleftarrow{\pi}(\alpha)=\left(\pi\left(\alpha^{R}\right)\right)^{R}, \overleftarrow{\lambda}(\alpha)=$ $\left(\lambda\left(\alpha^{R}\right)\right)^{R}, \overleftarrow{\varphi}(\alpha)=\left(\varphi\left(\alpha^{R}\right)\right)^{R}$ and $\overleftarrow{F}(\alpha)=\left(F\left(\alpha^{R}\right)\right)^{R}$

From the previous result and the fact that $\mathcal{A}_{\mathrm{pd}}(\alpha) \simeq \mathcal{A}_{\mathrm{pos}}(\alpha) / \equiv_{\mathrm{c}}$ we have

Proposition 3. For any $\alpha \in \mathrm{RE}$,

1. $\left(\mathcal{A}_{\mathrm{pd}}\left(\alpha^{R}\right)\right)^{R} \simeq \overleftarrow{\mathcal{A}}_{\mathrm{pd}}(\alpha)$

2. $\mathcal{L}\left(\overleftarrow{\mathcal{A}}_{\mathrm{pd}}(\alpha)\right)=\mathcal{L}(\alpha)$.

3. $\overleftarrow{\mathcal{A}}_{\mathrm{pd}}(\alpha) \simeq\left(\mathcal{A}_{\mathrm{pos}}\left(\alpha^{R}\right)\right)^{R} / \equiv_{c}$. 


\section{Prefix Automata}

Yamamoto [16] presented a new algorithm for converting a regular expression into an equivalent NFA. First, a labeled version of the usual Thompson NFA $(Q, \Sigma, \delta, I, F)$ is obtained, where each state $q$ is labeled with two regular expressions, one that corresponds to its left language, $L P(q)$, and the other to its right language, $L S(q)$. States which in-transitions are labeled with a letter are called sym-states. Then the equivalence relations $\equiv_{\text {pre }}$ and $\equiv_{\text {suf }}$ are defined on the set of sym-states: for two states $p, q \in Q, p \equiv_{\text {pre }} q$ if and only if $\operatorname{LP}(p)=$ $L P(q)$; and $p \equiv_{\text {suf }} q$ if and only if $L S(p)=L S(q)$. The prefix automaton $\mathcal{A}_{\text {pre }}$ and the suffix automaton $\mathcal{A}_{\text {suf }}$ are the quotient automata by these relations. The final automaton is a combination of these two. The author also shows that $\mathcal{A}_{\text {suf }}$ coincides with $\mathcal{A}_{\mathrm{pd}}$. This relation between $\mathcal{A}_{\mathrm{pd}}$ and $\mathcal{A}_{\text {suf }}$ could lead us to think that $\overleftarrow{\mathcal{A}}_{\mathrm{pd}}$ coincide with $\mathcal{A}_{\text {pre }}$, which is not true. For instance, considering $\alpha=a+b$, the $\overleftarrow{\mathcal{A}}_{\text {pd }}(\alpha)$ has 2 states and the $\mathcal{A}_{\text {pre }}(\alpha)$ has 3 states (see Fig. 2 ). Note that both automata are obtained from another automaton by merging the states with the same left language: while the $\overleftarrow{\mathcal{A}}_{\text {pd }}(\alpha)$ is obtained from $\left(\mathcal{A}_{\text {pos }}\left(\alpha^{R}\right)\right)^{R}$, we will see that the $\mathcal{A}_{\text {pre }}(\alpha)$ is obtained from $\mathcal{A}_{\text {pos }}(\alpha)$.

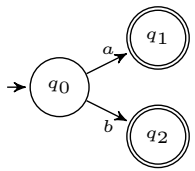

(a) $\mathcal{A}_{\text {pos }}(\alpha)$

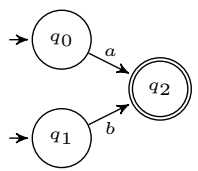

(b) $\left(\mathcal{A}_{\text {pos }}\left(\alpha^{R}\right)\right)^{R}$

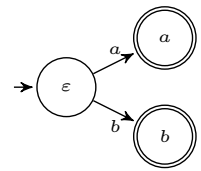

(c) $\mathcal{A}_{\text {pre }}(\alpha)$

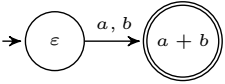

(d) $\overleftarrow{\mathcal{A}}_{\mathrm{pd}}(\alpha)$

Fig. 2. Automata for $\alpha=a+b$.

The $L P$ labelling scheme proposed by Yamamoto can be obtained as a solution of a system of expression equations for a $\operatorname{RE} \alpha$, as done both for $\mathcal{A}_{\text {pd }}$ and $\overleftarrow{\mathcal{A}}_{\mathrm{pd}}$. Consider a system of left equations $\alpha_{i}=\alpha_{i 1} \sigma_{1}+\cdots+\alpha_{i k} \sigma_{k}, i \in[1, n]$ where $\alpha=\sum_{i \in I \subseteq[0, n]} \alpha_{i}, \alpha_{i j}=\sum_{l \in I_{i j} \subseteq[0, n]} \alpha_{l}$ and $\alpha_{0} \sim \varepsilon$.

Proposition 4. The set $\operatorname{Pre}(\alpha)$ inductively defined as follows:

$$
\begin{aligned}
& \operatorname{Pre}(\emptyset)=\emptyset \quad \operatorname{Pre}(\alpha+\beta)=\operatorname{Pre}(\alpha) \cup \operatorname{Pre}(\beta) \\
& \operatorname{Pre}(\varepsilon)=\emptyset \quad \operatorname{Pre}(\alpha \beta)=\alpha \operatorname{Pre}(\beta) \cup \operatorname{Pre}(\alpha) \\
& \operatorname{Pre}(\sigma)=\{\sigma\} \quad \operatorname{Pre}\left(\alpha^{\star}\right)=\alpha^{\star} \operatorname{Pre}(\alpha) .
\end{aligned}
$$

is a solution (left support) of the system of left equations defined above.

The set $\operatorname{Pre}_{0}(\alpha)=\operatorname{Pre}(\alpha) \cup\{\varepsilon\}$ constitutes the set of states of the prefix automaton $\mathcal{A}_{\text {pre }}(\alpha)$. It also follows from the resolution of the above system of equations, that the set of transitions of $\mathcal{A}_{\text {pre }}(\alpha)$ can be inductively defined. Let $\mathrm{P}(\alpha), \psi(\alpha)$, and $\mathrm{T}(\alpha)$ be defined, respectively, as follows:

$$
\begin{aligned}
& \mathrm{P}(\emptyset)=\emptyset \\
& \mathrm{P}(\varepsilon)=\{\varepsilon\} \\
& \mathrm{P}(\alpha+\beta)=\mathrm{P}(\alpha) \cup \mathrm{P}(\beta) \\
& \mathrm{P}(\sigma)=\{\sigma\} \\
& \mathrm{P}(\alpha \beta)=\alpha \mathrm{P}(\beta) \cup \varepsilon(\beta) \mathrm{P}(\alpha) \\
& \mathrm{P}\left(\alpha^{\star}\right)=\alpha^{\star} \mathrm{P}(\alpha) \text {. }
\end{aligned}
$$




$$
\begin{array}{ccrl}
\psi(\emptyset)=\emptyset & \psi(\alpha+\beta) & =\psi(\alpha) \cup \psi(\alpha) \\
\psi(\varepsilon)=\emptyset & \psi(\alpha \beta) & =\psi(\alpha) \cup \varepsilon(\alpha) \alpha \psi(\beta) \\
\psi(\sigma)=\{(\sigma, \sigma)\} & \psi\left(\alpha^{\star}\right) & =\alpha^{\star} \psi(\alpha) \\
& & \\
\mathrm{T}(\emptyset) & =\mathrm{T}(\varepsilon)=\mathrm{T}(\sigma)=\emptyset, \sigma \in \Sigma \\
\mathrm{T}(\alpha+\beta) & =\mathrm{T}(\alpha) \cup \mathrm{T}(\beta) \\
\mathrm{T}(\alpha \beta) & =\mathrm{T}(\alpha) \cup \alpha \mathrm{T}(\beta) \cup \mathrm{P}(\alpha) \times(\alpha \psi(\beta)) \\
\mathrm{T}\left(\alpha^{\star}\right) & =\alpha^{\star} \mathrm{T}(\alpha) \cup \alpha^{\star}(\mathrm{P}(\alpha) \times \psi(\alpha)) .
\end{array}
$$

Therefore, $\mathcal{A}_{\text {pre }}(\alpha)=\left(\operatorname{Pre}_{0}(\alpha), \Sigma,\{\varepsilon\} \times \psi(\alpha) \cup \mathrm{T}(\alpha), \varepsilon, \mathrm{P}(\alpha) \cup \varepsilon(\alpha)\right)$. In Fig.3(b) we can see the $\mathcal{A}_{\text {pre }}(\beta b)$, where the RE $\beta b$ is the one of Fig. 1 . From both figures we observe that $\overleftarrow{\mathcal{A}}_{\text {pd }}(\beta b)$ is the smallest of the four automaton constructions. We

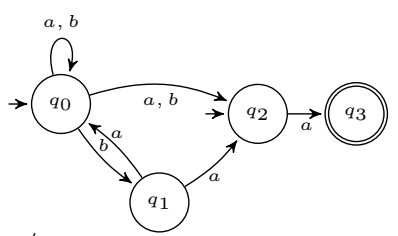

(a) $\overleftarrow{\mathcal{A}}_{\mathrm{pd}}(\beta b): q_{0}=\beta a^{\star}$ $q_{1}=\beta a^{\star} b, q_{2}=\beta, q_{3}=\beta b$

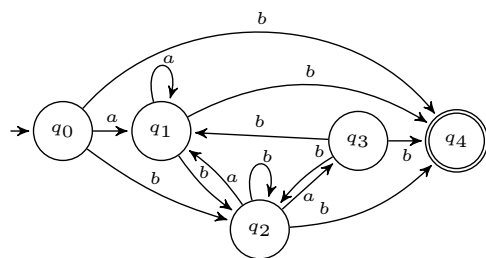

(b) $\mathcal{A}_{\text {pre }}(\beta b): q_{0}=\varepsilon, q_{1}=\beta\left(a^{\star} a\right)$, $q_{2}=\beta\left(a^{\star} b\right), q_{3}=\beta\left(\left(a^{\star} b\right) a\right)$, $q_{4}=\beta b$.

Fig. 3. Automata for $\beta b$, where $\beta=\left(a^{\star} b+a^{\star} b a+a^{\star}\right)^{\star}$

now show that the $\mathcal{A}_{\text {pre }}$ is a quotient of $\mathcal{A}_{\text {pos }}$. If $\alpha$ is a linear regular expression, $\mathcal{A}_{\text {pos }}(\alpha)$ is deterministic and thus all its states have distinct left languages. Therefore, in this case, $\mathcal{A}_{\text {pre }}(\alpha)$ coincides with $\mathcal{A}_{\text {pos }}(\alpha)$ and $|\operatorname{Pre}(\alpha)|=|\alpha|_{\Sigma}$. For an arbitrary $\operatorname{RE~} \alpha, \mathcal{A}_{\text {pre }}(\bar{\alpha}) \simeq \mathcal{A}_{\text {pos }}(\bar{\alpha})$. Let $\equiv_{l}$ be the equivalence relation in $\operatorname{Pre}(\bar{\alpha})$ such that for any regular expression $\alpha, \forall \alpha_{1}, \alpha_{2} \in \operatorname{Pre}(\bar{\alpha}), \alpha_{1} \equiv_{l} \alpha_{2} \Leftrightarrow \overline{\alpha_{1}}=\overline{\alpha_{2}}$. It is not difficult to see that $\equiv_{l}$ is a left-invariant relation.

Proposition 5. Let $\alpha$ be a regular expression. Then $\mathcal{A}_{\text {pre }}(\alpha) \simeq \mathcal{A}_{\text {pos }}(\alpha) / \equiv_{l}$.

By construction, the Glushkov automaton is homogeneous, i.e. the in- transitions of each state are all labelled by the same letter. It follows from Proposition 5 that this property also holds for $\mathcal{A}_{\text {pre }}$.

\section{Average-Case Complexity}

We conducted some experimental tests in order to compare the sizes of $\mathcal{A}_{\text {pos }}$, $\mathcal{A}_{\mathrm{pd}}, \overleftarrow{\mathcal{A}}_{\mathrm{pd}}$ and $\mathcal{A}_{\text {pre automata. We used the FAdo library }}{ }^{1}$ that includes implementations of the NFA conversions and also several tools for uniformly random generate regular expressions. In order to obtain regular expressions uniformly

\footnotetext{
${ }^{1}$ http://fado.dcc.fc.up.pt
} 
generated in the size of the syntactic tree, a prefix notation version of the grammar was used. For each alphabet size, $k$, and $|\alpha|$, samples of 10000 REs were generated, which is sufficient to ensure a $95 \%$ confidence level within a $1 \%$ error margin. Table 1 presents the average values obtained for $|\alpha| \in\{100,500,1000\}$ and $k \in\{2,10\}$. These experiments suggest that in pratice the $\overleftarrow{\mathcal{A}}_{\mathrm{pd}}$ and the

\begin{tabular}{|c|c|c|c|c|c|c|c|c|c|c|c|c|c|}
\hline$k$ & $|\alpha| \mid$ & || $\operatorname{pos}_{0} \mid$ & $\left|\delta_{\text {pos }}\right|$ & $|\mathrm{PD}| \mid$ & $\left|\delta_{\pi}\right|$ & $\mid \frac{|\pi|}{\mid \text { pos } \mid}$ & $|\overleftarrow{\mathrm{PD}}|$ & $\left|\delta_{\overleftarrow{\pi}}\right|$ & $\mid \frac{|\overleftarrow{\pi}|}{|\operatorname{pos}|}$ & $\left|\operatorname{Pre}_{0}\right| \mid$ & $\left|\delta_{\text {pre }}\right|$ & $\left|\frac{\mid \text { Pre| }}{\mid \text { |ps }}\right|$ & $1-\eta_{k}$ \\
\hline & \begin{tabular}{|c|}
100 \\
\end{tabular} & $\overline{28.9}$ & 167.5 & $\overline{|c|}$ & $\overline{56.0}$ & 0.55 & \begin{tabular}{|l|}
15.9 \\
\end{tabular} & $\overline{56.4}$ & 0.55 & 20.1 & 73.7 & \begin{tabular}{|l|}
0.71 \\
\end{tabular} & \multirow{2}{*}{0.90} \\
\hline 2 & 500 & 139.9 & 1486.5 & 71.6 & 389.8 & 0.51 & 71.5 & 393.1 & 0.51 & 91.9 & 530.8 & 0.66 & \\
\hline \multirow{3}{*}{10} & 100 & 42.5 & 159.4 & 23.8 & 73.7 & 0.56 & 23.8 & 72.9 & 0.56 & 38.5 & 130 & 0.91 & \multirow{3}{*}{0.99} \\
\hline & 500 & 207.1 & 1019.1 & 113.2 & 423.8 & $\mid 0.55$ & 112.4 & 425.6 & \begin{tabular}{|l|}
0.54 \\
\end{tabular} & 186 & 807.1 & 0.90 & \\
\hline & 000 & 2.1 & 182.1 & 223.7 & 884.1 & $\mid 0.54$ & 223.1 & 884.5 & $\mid 0.54$ & 369.5 & 717.6 & 0.90 & \\
\hline
\end{tabular}

Table 1. Experimental results for uniform random generated regular expressions.

$\mathcal{A}_{\mathrm{pd}}$ have the same size and the $\mathcal{A}_{\text {pre }}$ is not significantly smaller then the $\mathcal{A}_{\text {pos }}$. By Proposition 3, $\left|\alpha^{R}\right|_{\Sigma}=|\alpha|_{\Sigma}$ and by the fact that $\varepsilon \in \pi(\alpha)$ if and only if $\varepsilon \in \overleftarrow{\pi}(\alpha)$, the analysis of the average size of $\mathcal{A}_{\mathrm{pd}}(\alpha)$ presented in Broda et al [2] carries on to $\overleftarrow{\mathcal{A}}_{\mathrm{pd}}(\alpha)$. Thus the average sizes of $\mathcal{A}_{\mathrm{pd}}$ and $\overleftarrow{\mathcal{A}}_{\mathrm{pd}}$ are asymptotically the same. However, $\overleftarrow{\mathcal{A}}_{\mathrm{pd}}(\alpha)$ has only one final state and its number of initial states is the number of final states of $\mathcal{A}_{\mathrm{pd}}\left(\alpha^{R}\right)$. As studied by Nicaud [14], the size of last $(\alpha)$ tends asymptotically to a constant depending on $k$ and $|\lambda(\alpha)|$ is half that size [3]. Thus, that constant value will be also the number of initial states of $\overleftarrow{\mathcal{A}}_{\text {pd }}$. Following, again, the ideas in Broda et al., we estimate the number of mergings of states that arise when computing $\mathcal{A}_{\text {pre }}$ from $\mathcal{A}_{\text {pos }}$. The $\mathcal{A}_{\text {pre }}$ has at most $|\alpha|_{\Sigma}+1$ states and this only occurs when all unions in $\operatorname{Pre}(\alpha)$ are disjoint. However there are cases in which this does not happen. For instance, when $\sigma \in \operatorname{Pre}(\beta) \cap \operatorname{Pre}(\gamma)$, then $|\operatorname{Pre}(\beta+\gamma)|=|\operatorname{Pre}(\beta) \cup \operatorname{Pre}(\gamma)| \leq|\operatorname{Pre}(\beta)|+|\operatorname{Pre}(\gamma)|-1$ and $\left|\operatorname{Pre}\left(\beta^{\star} \gamma\right)\right|=\left|\beta^{\star} \operatorname{Pre}(\gamma) \cup \beta^{\star} \operatorname{Pre}(\beta)\right| \leq|\operatorname{Pre}(\beta)|+|\operatorname{Pre}(\gamma)|-1$. In what follows we estimate the number of these non-disjoint unions, which correspond to a lower bound for the number of states merged in the $\mathcal{A}_{\text {pos }}$ automaton. This is done in the framework of analytic combinatorics as expounded by Flajolet and Sedgewick [7]. The methods apply to generating functions $A(z)=\sum_{n} a_{n} z^{n}$ for a combinatorial class $\mathcal{A}$ with $a_{n}$ objects of size $n$, denoted by $\left[z^{n}\right] A(z)$, and also bivariate functions $C(u, z)=\sum_{\alpha} u^{c(\alpha)} z^{|\alpha|}$, where $c(\alpha)$ is some measure of the object $\alpha \in \mathcal{A}$.

The regular expressions $\alpha_{\sigma}$ for which $\sigma \in \operatorname{Pre}\left(\alpha_{\sigma}\right), \sigma \in \Sigma$, are generated by following grammar:

$$
\alpha_{\sigma}:=\sigma\left|\alpha_{\sigma}+\alpha\right| \alpha_{\bar{\sigma}}+\alpha_{\sigma}\left|\alpha_{\sigma} \cdot \alpha\right| \varepsilon \cdot \alpha_{\sigma}
$$

The regular expressions that are not generated by $\alpha_{\sigma}$ are denoted by $\alpha_{\bar{\sigma}}$. The generating function for $\alpha_{\sigma}, R_{\sigma, k}(z)$ satisfies

$$
\begin{aligned}
R_{\sigma, k}(z)= & z+z R_{\sigma, k}(z) R_{k}(z)+z\left(R_{k}(z)-R_{\sigma, k}(z)\right) R_{\sigma, k}(z)+ \\
& +z R_{\sigma, k}(z) R_{k}(z)+z^{2} R_{\sigma, k}(z)
\end{aligned}
$$


From this one gets

$$
R_{\sigma, k}(z)=\frac{\left(z^{2}+3 z R_{k}(z)-1\right)+\sqrt{\left(z^{2}+3 z R_{k}(z)-1\right)^{2}+4 z^{2}}}{2 z} .
$$

where $R_{k}(z)=\frac{1-z-\sqrt{\Delta_{k}(z)}}{4 z}$ is the generating function for REs given by gram$\operatorname{mar}(1)$ but omitting the $\emptyset, \Delta_{k}(z)=1-2 z-(7+8 k) z^{2}$ and following Nicaud,

$$
\left[z^{n}\right] R_{k}(z) \sim \frac{\sqrt{2\left(1-\rho_{k}\right)}}{8 \rho_{k} \sqrt{\pi}} \rho_{k}^{-n} n^{-3 / 2}, \text { where } \rho_{k}=\frac{1}{1+\sqrt{8 k+8}}
$$

Using the techniques in Broda et. al and namely Proposition 3 one has

$$
\left[z^{n}\right] R_{\sigma, k}(z) \sim \frac{3}{16 \sqrt{\pi}}\left(1-\frac{b\left(\rho_{k}\right)}{\sqrt{a\left(\rho_{k}\right)}}\right) \sqrt{2\left(1-\rho_{k}\right)} \rho_{k}^{-(n+1)} n^{-\frac{3}{2}},
$$

where $a(z)$ and $b(z)$ are polynomials. Thus, the asymptotic ratio of regular expressions with $\sigma \in \operatorname{Pre}(\alpha)$ is:

$$
\frac{\left[z^{n}\right] R_{\sigma, k}(z)}{\left[z^{n}\right] R_{k}(z)} \sim \frac{3}{2}\left(1-\frac{b\left(\rho_{k}\right)}{\sqrt{a\left(\rho_{k}\right)}}\right) .
$$

As $\lim _{k \rightarrow \infty} \rho_{k}=0, \lim _{k \rightarrow \infty} a\left(\rho_{k}\right)=1$, and $\lim _{k \rightarrow \infty} b\left(\rho_{k}\right)=1$, this asymptotic ratio tends to 0 with $k \rightarrow \infty$.

Let $i(\alpha)$ be the number of non-disjoint unions appearing during the computation of $\operatorname{Pre}(\alpha)$ originated by the two cases above. Then $i(\alpha)$ verifies

$$
\begin{array}{rlrl}
i(\varepsilon) & =i(\sigma)=0 & i\left(\alpha_{\sigma}^{\star} \alpha_{\sigma}\right) & =i\left(\alpha_{\sigma}^{\star}\right)+i\left(\alpha_{\sigma}\right)+1 \\
i\left(\alpha_{\sigma}+\alpha_{\sigma}\right) & =i\left(\alpha_{\sigma}\right)+i\left(\alpha_{\sigma}\right)+1 & i\left(\alpha_{\sigma}^{\star} \alpha_{\sigma}\right) & =i\left(\alpha_{\sigma}^{\star}\right)+i\left(\alpha_{\sigma}\right) \\
i\left(\alpha_{\sigma}+\alpha_{\bar{\sigma}}\right) & =i\left(\alpha_{\sigma}\right)+i\left(\alpha_{\bar{\sigma}}\right) & i\left(\alpha \alpha_{\bar{\sigma}}\right) & =i(\alpha)+i\left(\alpha_{\bar{\sigma}}\right) \\
i\left(\alpha_{\bar{\sigma}}+\alpha\right) & =i\left(\alpha_{\bar{\sigma}}\right)+i(\alpha) & i\left(\alpha^{\star}\right) & =i(\alpha) .
\end{array}
$$

From these equations we can obtain the cost generating function for the number of mergings:

$$
I_{\sigma, k}(z)=\frac{\left(z+z^{2}\right) R_{\sigma, k}(z)^{2}}{\sqrt{\Delta_{k}(z)}} .
$$

Using again the same Proposition 3 from Broda et al., we conclude that:

$$
\left[z^{n}\right] I_{\sigma, k}(z) \sim \frac{1+\rho_{k}}{64} \frac{\left(a\left(\rho_{k}\right)+b\left(\rho_{k}\right)^{2}-2 b\left(\rho_{k}\right) \sqrt{a\left(\rho_{k}\right)}\right)}{\sqrt{\pi} \sqrt{2-2 \rho_{k}}} \rho_{k}^{-(n+1)} n^{-\frac{1}{2}} .
$$

The cost generating function for the number of letters in $\alpha \in \mathrm{RE}$, computed by Nicaud, is $L_{k}(z)=\frac{k z}{\sqrt{\Delta_{k}(z)}}$ and $\left[z^{n}\right] L_{k}(z) \sim \frac{k \rho_{k}}{\sqrt{\pi\left(2-2 \rho_{k}\right)}} \rho_{k}^{-n} n^{-1 / 2}$. With these, we get an asymptotic estimate for the average number of mergings given by:

$$
\frac{\left[z^{n}\right] I_{\sigma, k}(z)}{\left[z^{n}\right] L_{k}(z)} \sim \frac{1-\rho_{k}}{4 \rho_{k}^{2}} \lambda_{k}=\eta_{k},
$$


where $\lambda_{k}=\frac{\left(1+\rho_{k}\right)}{16\left(1-\rho_{k}\right)}\left(a\left(\rho_{k}\right)+b\left(\rho_{k}\right)^{2}-2 b\left(\rho_{k}\right) \sqrt{a\left(\rho_{k}\right)}\right)$. It is not difficult to conclude that $\lim _{k \rightarrow \infty} \lambda_{k}=0$, therefore $\lim _{k \rightarrow \infty} \eta_{k}=0$. As it is evident from the last two columns of Table 1 , for small values of $k$, the lower bound $\eta_{k}$ does not capture all the mergings that occur in $\mathcal{A}_{\text {pre }}$. Although we must study other contributions for those mergings, it seems that for larger values of $k$, the average number of states of the $\mathcal{A}_{\text {pre }}$ automaton approaches the number of states of the $\mathcal{A}_{\text {pos }}$ automaton.

\section{References}

1. Antimirov, V.M.: Partial derivatives of regular expressions and finite automaton constructions. Theor. Comput. Sci. 155(2), 291-319 (1996)

2. Broda, S., Machiavelo, A., Moreira, N., Reis, R.: On the average state complexity of partial derivative automata. Int. J. Found. Comput. Sci. 22(7), 1593-1606 (2011)

3. Broda, S., Machiavelo, A., Moreira, N., Reis, R.: On the average size of Glushkov and partial derivative automata. Int. J. Found. Comput. Sci. 23(5), 969-984 (2012)

4. Champarnaud, J.M., Dubernard, J.P., Jeanne, H., Mignot, L.: Two-sided derivatives for regular expressions and for Hairpin expressions. In: Dediu, A.H., MartínVide, C., Truthe, B. (eds.) 7th LATA. LNCS, vol. 7810, pp. 202-213. Springer (2013)

5. Champarnaud, J.M., Ziadi, D.: From Mirkin's prebases to Antimirov's word partial derivatives. Fundam. Inform. 45(3), 195-205 (2001)

6. Champarnaud, J.M., Ziadi, D.: Canonical derivatives, partial derivatives and finite automaton constructions. Theor. Comput. Sci. 289(1), 137-163 (2002)

7. Flajolet, P., Sedgewick, R.: Analytic Combinatorics. CUP (2008)

8. Giammarresi, D., Ponty, J.L., Wood, D.: The Glushkov and Thompson constructions: a synthesis. unpublished manuscript (1998)

9. Glushkov, V.M.: The abstract theory of automata. Russian Mathematical Surveys 16(5), 1-53 (1961)

10. Ilie, L., Yu, S.: Follow automata. Inf. Comput. 186(1), 140-162 (2003)

11. Ko, S., Han, Y.: Left is better than right for reducing nondeterminism of nfas. In: Holzer, M., Kutrib, M. (eds.) 19th CIAA. LNCS, vol. 8587, pp. 238-251. Springer (2014)

12. Maia, E., Moreira, N., Reis, R.: Partial derivative and position bisimilarity automata. In: Holzer, M., Kutrib, M. (eds.) 19th CIAA. LNCS, vol. 8587, pp. 264277. Springer (2014)

13. Mirkin, B.: An algorithm for constructing a base in a language of regular expressions. Engineering Cybernetics 5, 110-116 (1966)

14. Nicaud, C.: On the average size of Glushkov's automata. In: Dediu, A.H., Ionescu, A.M., Martín-Vide, C. (eds.) 3rd LATA. LNCS, vol. 5457, pp. 626-637. Springer (2009)

15. Thompson, K.: Regular expression search algorithm. Com. ACM 11(6), 410-422 (1968)

16. Yamamoto, H.: A new finite automaton construction for regular expressions. In: Bensch, S., Freund, R., Otto, F. (eds.) NCMA. books@ocg.at, vol. 304, pp. 249264. Österreichische Computer Gesellschaft (2014) 